\title{
CD147 Immunohistochemistry Discriminates Between Reactive Mesothelial Cells and Malignant Mesothelioma
}

Céline Pinheiro, PH.D., ${ }^{1,2^{*}}$ Adhemar Longatto-Filho, PH.D., PMIAC, ${ }^{1,2,3}$

Tony R. Soares, M.sc., ${ }^{1,2}$ Helena Pereira, M.Sc., ${ }^{4}$

Carlos Bedrossian, M.D., PH.D. (HON), FIAC ${ }^{5}$ Claire Michael, M.D., ${ }^{6}$

Fernando C. Schmitt, M.D., PH.D., FIAC, ${ }^{7,8}$ and Fátima Baltazar, PH.D. ${ }^{1,2}$

Malignant mesothelioma (MM) is a rare form of cancer. Its histopathological diagnosis is very difficult, as it exhibits a number of different appearances that can be misinterpreted as metastatic invasion or atypical hyperplasia. Thus, there is an urgent need to identify adequate markers to distinguish between benign and malignant cells, allowing the implementation of appropriate therapies and, possibly, specific directed therapies. MM, like other tumors, show an increase in glucose uptake, due to high rates of glycolysis, inducing an intracellular overload of acids. In this context, monocarboxylate transporters (MCTs) emerge as important players, by mediating the transmembranar co-transport of lactate with a proton, thereby, regulating $\mathrm{pH}$ and allowing continuous glycolysis. Importantly, proper MCT expression and activity depend on its coexpression with a chaperone, CD147, which is associated with poor prognosis in cancer. Twenty-two samples including reactive

${ }^{1}$ Life and Health Sciences Research Institute, Health Sciences School, University of Minho, Braga, Portugal

${ }^{2}$ ICVS/3B's-PT Government Associate Laboratory, Braga/Guimarães, Portugal

${ }^{3}$ Laboratory of Medical Investigation 14, Faculty of Medicine, University of São Paulo, São Paulo, Brazil

${ }^{4}$ Centre of Molecular and Environmental Biology, University of Minho, Braga, Portugal

${ }^{5}$ Department of Pathology, Rush University Medical College, Chicago, Illinois

'Department of Cytopathology, University of Michigan, Ann Arbor, Michigan

${ }^{7}$ Medical Faculty, University of Porto, Porto, Portugal

${ }^{8}$ Institute of Molecular Pathology and Immunology of University of AQ3 Porto, Porto, Portugal

*Correspondence to: Céline Pinheiro, PH.D., Life and Health Sciences Research Institute, School of Health Sciences, University of Minho,

4710-057 Braga, Portugal. E-mail: cpinheiro@ecsaude.uminho.pt

Received 14 November 2011; Accepted 19 November 2011

DOI $10.1002 /$ dc. 22821

Published online in Wiley Online Library (wileyonlinelibrary.com). mesothelial cells, MM, and atypical mesothelial hyperplasias were evaluated for immunoexpression of MCT1, MCT4, and CD147. Expression of these proteins was compared with GLUT1 as a new promising marker for MM. Although MCT isoforms were not differentially expressed in the two types of cytological specimens CD147, as GLUT1, was almost exclusively expressed in MM. Both MCT1 and MCT4 are not able to discriminate between mesothelial reactive cells and mesothelial malignant cells, while CD147 was able to distinguish these two proliferations. If confirmed, besides being a good marker for identification of MM, CD147 may also be a target for therapeutical strategies in this rare type of tumor. Diagn. Cytopathol. 2011;00:000-000. @ 2011 Wiley Periodicals, Inc.

Key Words: monocarboxylate transporters; CD147/EMMPRIN; malignant mesothelioma; lactate

Malignant mesothelioma (MM), a neoplasm of mesothelial cells from the serosal membranes occurs predominantly in the pleura, less often in the peritoneum, seldom in pericardium, and rarely in the tunica vaginalis testis. Asbestos exposure is the most common cause of MM and its connection with this rare form of cancer has been well established for the last half a century. ${ }^{1}$ The long-latency period from initial asbestos exposure to development of MM is responsible for an incidence of nearly 3,000 new cases a year in the U.S., a number that may remain at that level for the foreseeable future. ${ }^{2}$ The number of $\mathrm{MM}$ diagnosed worldwide may also grow larger due to pathologists' increasing ability in recognizing $\mathrm{MM}$ and to the emergence of newer immunohistochemical markers with restrictive specificity for this neoplasm.

Current MM treatment is complex and the results are far from ideal. Surgery cannot remove all the residual mi- 
croscopic cancer deposits and as a result, surgical treatment for operable MM even in able hands cannot be considered a definite form of therapy. ${ }^{3}$ Even with new forms of systemic therapy, survival beyond 12-18 months is extremely rare. ${ }^{4}$ Attempts at combined chemotherapy, ${ }^{5}$ gene therapy, ${ }^{6}$ and immunotherapy with vaccines ${ }^{7}$ have not fared any better. The attention, therefore, has shifted to targeted therapy directed at molecular events involved in the survival and growth of malignant transformed mesothelial cells. Specific-targeted therapy has been a success story in the treatment of GIST and other tumors and constitutes a promising option for the treatment of MM. ${ }^{8}$

A precise pathological diagnosis of $\mathrm{MM}$ remains crucial for instituting appropriate therapeutic intervention. Early diagnosis offers the best opportunity for successful outcomes in any treatment strategy and cytological examination of effusion specimens offers the greatest chance for achieving it. However, in its early stage, the distinction between MM and atypical mesothelial hyperplasia still represents a very difficult challenge. ${ }^{9}$ Cytological results have vastly improved with the use of newer antibody panels but no infallible marker has been developed to distinguish reactive mesothelial hyperplasia from MM. ${ }^{10,11}$ More recently, the combination of positive epithelial membrane antigen (EMA) and negative desmin was interpreted to strongly favor MM, whereas a combination of negative EMA and positive desmin favors a reactive process. ${ }^{12}$

Some new promising markers have emerged recently, including some like GLUT1, ${ }^{13}$ which play a role in cellular glycolytic metabolism. The expression of GLUT1 may even discriminate between reactive mesothelium and $\mathrm{MM},{ }^{13}$ although with a lower specificity and sensitivity than originally claimed. ${ }^{14}$ Following this line of evidence, some studies suggest that positron emission tomography with ${ }^{18} \mathrm{~F}$-fluorodeoxyglucose (FDG-PET) imaging could be an effective tool for differentiating between benign and malignant pleural diseases, ${ }^{15-17}$ indicating a higher glycolytic metabolism in the malignant context. It is widely known that, under hypoxic conditions, cancer cells obtain energy by increasing their rates of glycolysis leading to an overload of intracellular lactic acid, which must be transported out of the cell, a process that results in extracellular acidification. Low interstitial $\mathrm{pH}$ is associated with the upregulation of various angiogenic molecules such as VEGF, IL-8, bFGGF, and Heparanase, all of which may provide autocrine stimulation and contribute to tumor aggressiveness. ${ }^{18}$ Cancer lactate accumulation has also been correlated with poor clinical outcomes, ${ }^{19-21}$ a property that remains yet to be explored as part of tumoral treatment strategies. One of the most important players in this context is monocarboxylate transporters (MCTs), which are also responsible for transmembrane co-transport of lactate with a proton, thus regulating
$\mathrm{pH}$ and enabling continuous glycolysis. The authors have observed that some MCT isoforms (MCT1 and MCT4) and their chaperone CD147 are overexpressed in a variety of solid tumors, including cervical carcinoma, ${ }^{22,23}$ colorectal carcinoma, ${ }^{24}$ and basal-like carcinoma of the breast $^{25}$ but not in gastric carcinoma. ${ }^{26}$ Since MCT activity supports cancer cell survival, their capacity to transport lactate can be explored as a new therapeutic target. In addition, CD147, the MCT1/MCT4 chaperone, was shown to contribute to tumoral progression and metastasis. Therefore, the metabolic modification of the tumor's microenvironment mediated by MCT1/CD147 holds promise as a target for future therapeutic options. ${ }^{27} \mathrm{De}-$ spite their potential as molecular therapeutic targets for neoplasms relying on glycolytic metabolism, MCTs have not been investigated in MM. The aim of this study was to characterize the expression of monocarboxylate transporters 1 and 4 and their chaperone CD147 in benign and malignant mesothelial cells, and compare their expression with GLUT1, a new promising MM marker.

\section{Material and Methods}

Samples were obtained from 20 patients including 11 mesothelial reactive cells and $9 \mathrm{MM}$. Samples were retrieved from the consultation files of one of the authors (CWB). Relevant data available included patient's age and gender, source of the effusion as well as the final histopathological diagnosis in pleural biopsies or decortication specimens.

\section{Immunohistochemistry}

MCT, CD147, and GLUT1 detection. MCT immunohistochemistry was performed according to the avidin-biotin-peroxidase complex method (R.T.U. VECTASTAIN Elite ABC Kit (Universal), Vector Laboratories, Burlingame, CA), with primary antibodies for MCT1 (AB3538P, Chemicon International, Temecula, CA), and MCT4 (AB3316P, Chemicon International, Temecula, CA), diluted 1:200, as previously described. ${ }^{24}$ Immunohistochemistry for CD147 and GLUT1 was performed according to the streptavidin-biotin-peroxidase complex principle Ultravision Detection System Anti-polyvalent, HRP, Lab Vision Corporation, Fremont, CA), using primary antibodies raised against CD147 (18-7344, ZYMED Laboratories Inc., South San Francisco, CA, diluted 1:750, as previously described ${ }^{23}$ ) and GLUT1 (ab15309, AbCam, Cambridge, UK, diluted 1:500, same protocol as for CD147). Negative controls were performed using appropriate serum controls for the primary antibodies (N1699, Dako, Carpinteria, CA), colon carcinoma tissue was used as positive control for both MCT1 and MCT4, cervical squamous carcinoma for CD147 and skin for GLUT1. Tissue sections were counterstained with hematoxylin and permanently mounted. 
Table 1. Immunohistochemical Characterization of MCT1, MCT4, CD147, and GLUT1 in 20 Patients with Mesothelioma and Benign Mesothelial Reaction

\begin{tabular}{|c|c|c|c|c|c|c|c|c|c|}
\hline Case & Gender & Age & $\begin{array}{c}\text { Histopathological } \\
\text { diagnosis }\end{array}$ & $\begin{array}{l}\text { MCT1 } \\
\text { score }\end{array}$ & $\begin{array}{c}\text { MCT1 } \\
\text { membrane }\end{array}$ & $\begin{array}{c}\text { MCT4 } \\
\text { score }\end{array}$ & $\begin{array}{l}\text { CD147 } \\
\text { score }\end{array}$ & $\begin{array}{c}\text { CD147 } \\
\text { membrane }\end{array}$ & $\begin{array}{c}\text { GLUT1 } \\
\text { membrane }\end{array}$ \\
\hline 1 & Male & 82 & Mesothelioma & + & + & + & + & + & + \\
\hline 2 & Male & 70 & Benign & + & + & + & - & - & - \\
\hline 3 & Male & 80 & Mesothelioma & + & + & - & + & + & + \\
\hline 4 & Male & 47 & Benign & - & - & + & - & - & - \\
\hline 5 & Male & 69 & Mesothelioma & + & + & + & + & + & + \\
\hline 6 & Male & 78 & Mesothelioma & + & + & + & + & + & + \\
\hline 7 & Female & 78 & Benign & + & + & + & - & - & - \\
\hline 8 & Male & 82 & Benign & + & + & - & n.a. & n.a. & - \\
\hline 9 & Male & 81 & Mesothelioma & + & + & - & + & + & + \\
\hline 10 & Male & 81 & Benign & + & + & + & + & + & - \\
\hline 11 & Male & 58 & Benign & - & - & + & - & - & - \\
\hline 12 & Female & 72 & Mesothelioma & + & + & + & + & + & + \\
\hline 13 & Male & 69 & Benign & + & + & - & - & - & - \\
\hline 14 & Male & 76 & Benign & + & + & - & - & - & - \\
\hline 15 & Female & 52 & Benign & + & - & - & + & - & - \\
\hline 16 & Male & 62 & Benign & + & + & + & - & - & - \\
\hline 17 & Male & 89 & Benign & + & + & + & - & - & + \\
\hline 18 & Male & 55 & Mesothelioma & + & + & + & + & + & + \\
\hline 19 & Female & 73 & Mesothelioma & + & + & + & - & - & + \\
\hline 20 & Male & 55 & Mesothelioma & + & + & - & + & + & - \\
\hline
\end{tabular}

Benign means hyperplastic reactive mesothelia.n.a., not available.

Immunohistochemical evaluation. Sections were scored semi-quantitatively for immunoreaction as follows: 0: $0 \%$ of immunoreactive cells; $1:<5 \%$ of immunoreactive cells; 2: $5-50 \%$ of immunoreactive cells; and 3: $>50 \%$ of immunoreactive cells. Also, intensity of staining was scored semi-qualitatively as follows: 0: negative; 1: weak; 2: intermediate; and 3: strong. The final score was defined as the sum of both parameters (extent and intensity), and grouped as negative (score 0 and 2) and positive (score 3-6), as previously described. ${ }^{24}$ Since plasma membrane location of these proteins is essential for activity, when present, the significance of plasma membrane positivity for MCTs, CD147, and GLUT1 was evaluated separately. Immunohistochemical evaluation was performed blindly by two independent observers and discordant cases were discussed using a double-head microscope to determine the final score.

\section{Statistical Analysis}

Data were stored and analyzed using the SPSS statistical software (version 16.0, SPSS, Chicago, IL). All comparisons were examined for statistical significance using Pearson's chi-square $\left(\chi^{2}\right)$ test and Fisher's exact test (when $n$ $<5$ ), being threshold for significance $P$ values $<0.05$.

\section{Results}

Twenty samples from 16 male and 4 female patients were assessed for MCT1, MCT4, CD147, and GLUT1 immunohistochemical expression. The results obtained for each

T1 case are summarized in Table I. Positive MCT1 expression was observed in both plasma membrane and cyto-

F1 plasm (Figs. 1A and 1E), while MCT4 was only found in the cytoplasm (Figs. 1B and 1F). Regarding CD147, except for one case, expression was always present in the plasma membrane (Fig. 1G), with some cytoplasmic staining. GLUT1 expression was present in the plasma membrane of all positive cases (Fig. 1H), showing also a strong plasma membrane expression in red blood cells (Fig. 1D).

Comparison between the expression frequencies of all markers in MM cells and mesothelial reactive cells is depicted in Table II. No statistically significant difference was observed between the benign and malignant samples with regards to their MCT1 and MCT4 expression. In contrast, the expression of CD147 was significantly increased in the MM samples, being expressed in about $90 \%(8 / 9)$ of MM vs. 9\% (1/11) for mesothelial reactive cells $(P=0.001)$. The power of CD147 to distinguish benign from malignant mesothelial cells was the same as that of GLUT1, with about $90 \%$ of positivity for MM vs. $9 \%$ for mesothelial reactive cells $(P=0.001)$, corresponding to a sensitivity of $88.8 \%$ and specificity of $90.9 \%$. No associations were found between either of the two MCTs, CD147, or GLUT1 and age or gender (data not shown). In addition, MCTs were not significantly coexpressed with CD147 (data not shown).

\section{Discussion}

Appropriate therapeutic intervention in MM can only be achieved if an accurate pathological diagnosis is made. However, the distinction between MM and atypical mesothelial hyperplasia at early stages remains very difficult. ${ }^{9}$ Therefore, there is an urgent need to establish efficient markers to distinguish between these two cytological entities. 


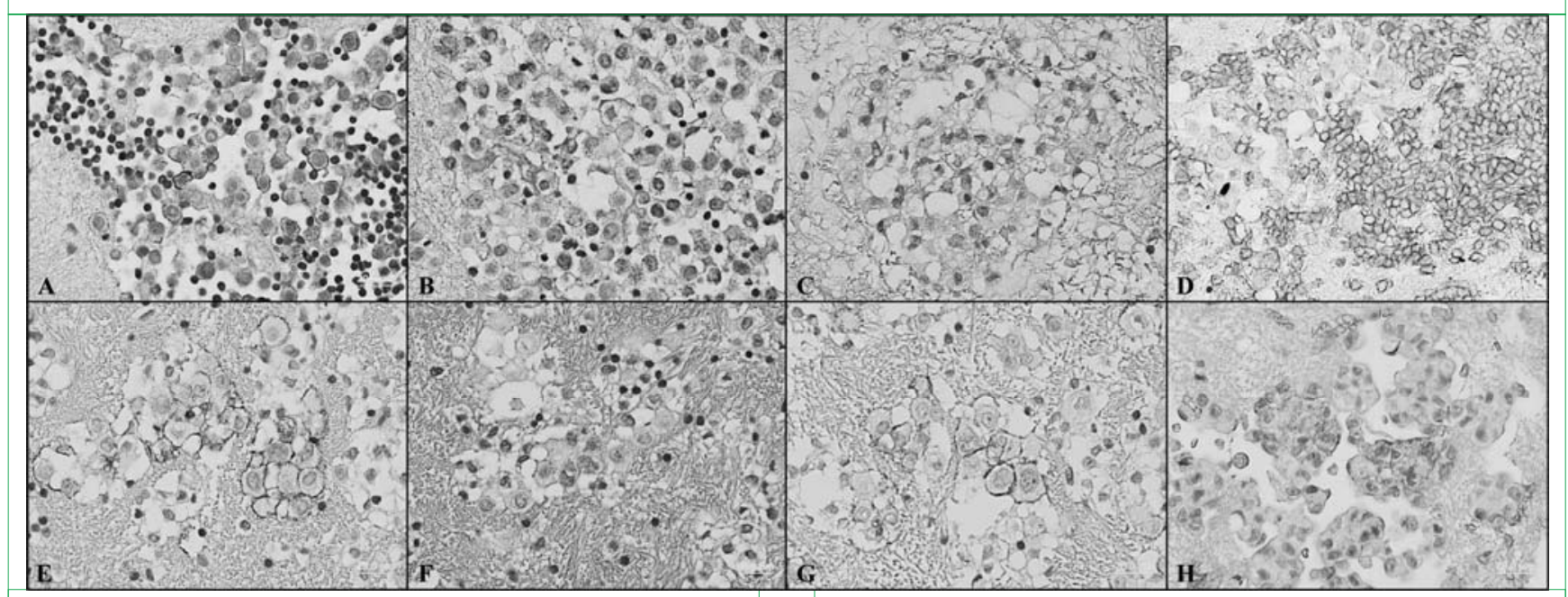

Fig. 1. Immunohistochemical expression of monocarboxylate transporter 1 (MCT1), MCT4, CD147, and glucose transporter 1 (GLUT1) in mesothelial samples. (A) MCT1 in benign mesothelial cells; (B) MCT4 in benign mesothelial cells; (C) CD147 in benign mesothelial cells; (D) GLUT1 in benign mesothelial cells; (E) MCT1 in malignant mesothelial cells; (F) MCT4 in malignant mesothelial cells; (G) CD147 in malignant mesothelial cells; (H) GLUT1 in malignant mesothelial cells. Note the strong expression of GLUT1 in the plasma membrane of red blood cells. [Color figure can be viewed in the online issue, which is available at wileyonlinelibrary.com.]

To the best of our knowledge, the results in this study reported described for the first time the expression of monocarboxylate transporters 1 and 4 in MM. However, neither MCT1 nor MCT4 showed any significant discriminating value in the recognition of malignancy. Actually, the values observed show a very similar pattern between mesothelial-derived malignancy and benign cells. Of note, MCT4 positive reaction was predominantly found in the cytoplasm which can explain, in part, the lack of significance of MCT4 in MM. Conversely, despite detection of MCT1 within the plasma membrane, MCT1 reaction did not show significant differences either. The overexpression of monocarboxylate transporters in both reactive mesothelium and MM supports the intriguing notion that benign mesothelial proliferations may be a precursor lesion of MM. In fact, the evolution of atypical mesothelial hyperplasia to invasive peritoneal MM has been documented, 8 years from the original non-MM diagnosis. ${ }^{28}$ Unfortunately, no laparoscopy or biopsy was available in the intervening years, to document or dispel the presence of an in situ stage.

CD147, also known as extracellular matrix metalloproteinase inducer (EMMPRIN), is recognized to promote tumor angiogenesis mostly through its protease-inducing function. Remarkably, the plasma membrane expression of CD147 was associated with MM, since the majority of MM cases were positive for CD147 and only 1 case of mesothelial hyperplasia was decorated by CD147 immunoreaction. This fact may be important on two different bases: first, membranous positive reaction for CD147 revealed to be a reliable marker of MM; second, it could be considered as a promising candidate for specific-targeted therapy. A more recent study revealed that CD147
Table II. Frequency of MCT1, MCT4, CD147, and GLUT1 Expressions in Mesothelioma Compared to Mesothelial Reactive Cells

\begin{tabular}{|lrlc|}
\hline & $n$ & Positive (\%) & $P$ \\
\hline MCT1 (membrane) & & & 0.218 \\
Mesothelial reactive cells & 11 & $8(72.7)$ & \\
Mesothelioma & 9 & $9(100.0)$ & 1.000 \\
MCT4 (cytoplasm) & & $7(63.6)$ & \\
Mesothelial reactive cells & 11 & $7(66.7)$ & 0.001 \\
Mesothelioma & 9 & $1(9.1)$ & \\
CD147 (membrane) & & $8(88.9)$ & 0.001 \\
Mesothelial reactive cells & 11 & $1(9.1)$ & \\
Mesothelioma & 9 & $8(88.9)$ & \\
GLUT1 (membrane) & 11 & & \\
Mesothelial reactive cells & 9 & & \\
Mesothelioma & & & \\
\hline \hline
\end{tabular}

may directly contribute to the angiogenic process regulation by association with upregulation of HIF- $2 \alpha$, VEGFR2 , and the soluble forms of VEGF in endothelial cells. ${ }^{29}$ This finding corroborated, in part, our previous report, where we found that VEGFR-3, a lymphatic vessel receptor which may be highly expressed in tumor cells and blood vessels of certain malignancies, was overexpressed in $\mathrm{MM}^{30}$ Importantly, these results also suggest that, in addition to increasing protease production, CD147 may contribute to the upregulation of soluble forms of VEGF in endothelial cells, thus directly regulating the angiogenic process. However, CD147 was not found to be accurate in predicting $\mathrm{MM}$ aggressiveness. ${ }^{31}$

MM responds unpredictably to therapy and treatment results seem to be related to biological properties, rather than to cytological grading or clinical staging. Molecular markers provide evergrowing insight into the biology of $\mathrm{MM}$, but fall short of being prognostic factors or as gages 
of treatment response. Currently, GLUT1 appears promising for guiding therapeutic strategies and evaluating their effectiveness. The strong GLUT1 expression the authors observed in MM confirms the experience of other investigators and suggests that MM has a preference for glycolysis. ${ }^{13}$ This property has been utilized in positron emission tomography (PET) in the evaluation of response to therapy. ${ }^{32}$ Based on these prior observations one would expect that either MCT1 or MCT4 would be upregulated in $\mathrm{MM}$, but this was not the case in our study. In fact, both MCT1 and MCT4 were equally positive in MM and mesothelial hyperplasia suggesting that these molecules behave as other proliferative markers that are positive in these two settings but cannot discriminate between them. On the other hand, the immunopositivity for CD147 paralleled the results of GLUT1, supporting the notion that CD147 is a valuable discriminator between benign and malignant mesothelial proliferations. In addition, we found that CD147 positive reactions were easier to interpret, since this marker did not stain red blood cells, as is the case with GLUT1 in the study of bloody effusions. Just as hyperthermic intraperitoneal chemotherapy enhances the results of cytoreductive surgery, ${ }^{33}$ it seems plausible to assume that altering the $\mathrm{pH}$ in the microenvironment of MM may enhance other modalities of therapy. This possibility has not been investigated.

In this study, although MCT expression was not increased in MM, when compared with reactive mesothelial cells, CD147 was found to discriminate between these two cytological entities, in a similar method to that described for GLUT1. However, our current findings deserve further study to substantiate their validity and to explore the potential use of CD147 as a factor in novel treatment strategies.

\section{Ethics}

The present study has been approved by the local Ethic Committees.

\section{Acknowledgments}

$\mathrm{CP}$ received a post-doctoral fellowship from FCT (Portuguese Foundation for Science and Technology, SFRH/ BPD/69479/2010).

\section{References}

1. Bedrossian CWM. Asbestos-related diseases - A historical and mineralogic perspective - Introduction. Semin Diagn Pathol 1992;9:9196.

2. Grondin SC, Sugarbaker DJ. Malignant mesothelioma of the pleural space. Oncology (New York) 1999;13:919-926.

3. Sugarbaker DJ, Wolf AS. Surgery for malignant pleural mesothelioma. Expert Rev Respir Med 2010;4:363-372.

4. Ray M, Kindler HL. Malignant pleural mesothelioma: an update on biomarkers and treatment. Chest 2009;136:888-896.
5. Bertino P, Carbone M, Pass H. Chemotherapy of malignant pleural mesothelioma. Expert Opin Pharmacother 2009;10:99-107.

6. van der Most RG, Robinson BWS, Nelson DJ. Gene therapy for malignant mesothelioma: Beyond the infant years. Cancer Gene Ther 2006;13:897-904.

7. Nowak AK, Lake RA, Kindler HL, Robinson BWS. New approaches for mesothelioma: Biologics, vaccines, gene therapy, and other novel agents. Semin Oncol 2002;29:82-96.

8. Pasello G, Favaretto A. Molecular targets in malignant pleural mesothelioma treatment. Curr Drug Targets 2009;10:1235-1244.

9. Bedrossian CWM. Diagnostic problems in serous effusions. Diagn Cytopathol 1998;19:131-137.

10. Bedrossian CWM. Special stains, the old and the new: The impact of immunocytochemistry in effusion cytology. Diagn Cytopathol 1998; 18:141-149.

11. Lyons-Boudreaux V, Mody DR, Zhai J, Coffey D. Cytologic malignancy versus benignancy - How useful are the "Newer" markers in body fluid cytology? Arch Pathol Lab Med 2008;132:23-28.

12. Hasteh F, Lin GY, Weidner N, Michael CW. The use of immunohistochemistry to distinguish reactive mesothelial cells from malignant mesothelioma in cytologic effusions. Cancer Cytopathol 2010;118:90-96.

13. Kato Y, Tsuta K, Seki K, Maeshima AM. Immunohistochemical detection of GLUT-1 can discriminate between reactive mesothelium and malignant mesothelioma. Mod Pathol 2007;20:215-220.

14. Shen J, Pinkus GS, Deshpande V, Cibas ES. Usefulness of EMA, GLUT-1, and XIAP for the cytologic diagnosis of malignant mesothelioma in body cavity fluids. Am J Clin Pathol 2009;131:516 523.

15. Bury T, Paulus P, Dowlati A, Corhay JL, Rigo P, Radermecker MF. Evaluation of pleural diseases with FDG-PET imaging: Preliminary report. Thorax 1997;52:187-189.

16. Carretta A, Landoni C, Melloni G. 18-FDG positron emission tomography in the evaluation of malignant pleural diseases - A pilot study. Eur J Cardiothorac Surg 2000;17:377-383.

17. Benard F, Sterman D, Smith RJ, Kaiser LR, Albelda SM, Alavi A. Metabolic imaging of malignant pleural mesothelioma with fluorodeoxyglucose positron emission tomography. Chest 1998;114:713-722.

18. Davidson B, Vintman L, Zcharia E, Bedrossian C, Berner A, Nielsen $S$, Ilan N, Vlodavsky I, Reich R. Heparanase and basic fibroblast growth factor are co-expressed in malignant mesothelioma. Clin Exp Metastasis 2004;21:469-476.

19. Brizel DM, Schroeder T, Scher RL. Elevated tumor lactate concentrations predict for an increased risk of metastases in head-and-neck cancer. Int J Radiat Oncol Biol Phys 2001;51:349-353.

20. Quennet V, Yaromina A, Zips D. Tumor lactate content predicts for response to fractionated irradiation of human squamous cell carcinomas in nude mice. Radiother Oncol 2006;81:130-135.

21. Walenta S, Wetterling M, Lehrke M. High lactate levels predict likelihood of metastases, tumor recurrence, and restricted patient survival in human cervical cancers. Cancer Res 2000;60:916-921.

22. Pinheiro C, Longatto-Filho A, Ferreira L. Increasing expression of monocarboxylate transporters 1 and 4 along progression to invasive cervical carcinoma. Int J Gynecol Pathol 2008;27:568-574.

23. Pinheiro C, Longatto A, Pereira SMM. Monocarboxylate transporters 1 and 4 are associated with CD147 in cervical carcinoma. Dis Markers 2009;26:97-103.

24. Pinheiro C, Longatto-Filho A, Scapulatempo C. Increased expression of monocarboxylate transporters 1,2 , and 4 in colorectal carcinomas. Virchows Arch 2008;452:139-146.

25. Pinheiro C, Albergaria A, Paredes J. Monocarboxylate transporter 1 is up-regulated in basal-like breast carcinoma. Histopathology 2010;56:860-867.

26. Pinheiro C, Longatto-Filho A, Simoes K. The prognostic value of CD147/EMMPRIN is associated with monocarboxylate transporter 1 co-expression in gastric cancer. Eur J Cancer 2009;45:2418-2424.

27. Kennedy KM, Dewhirst MW. Tumor metabolism of lactate: the influence and therapeutic potential for MCT and CD147 regulation. Future Oncol 2010;6:127-148. 


\section{Diagnostic Cytopathology DOI 10.1002/dc}

PINHEIRO ET AL.

28. Scurry J, Duggan MA. Malignant mesothelioma eight years after a diagnosis of atypical mesothelial hyperplasia. J Clin Pathol 1999; 52:535-537.

29. Bougatef F, Quemener C, Kellouche S. EMMPRIN promotes angiogenesis through hypoxia-inducible factor-2alpha-mediated regulation of soluble VEGF isoforms and their receptor VEGFR-2. Blood 2009; 114:5547-5556.

30. Filho AL, Baltazar F, Bedrossian C, Michael C, Schmitt FC. Immunohistochemical expression and distribution of VEGFR-3 in malignant mesothelioma. Diagn Cytopathol 2007;35:786-791.
31. Lumb PD, Suvarna SK. Metastasis in pleural mesothelioma. Immunohistochemical markers for disseminated disease. Histopathology 2004;44:345-352.

32. Ceresoli GL, Chiti A, Zucali PA. Early response evaluation in malignant pleural mesothelioma by positron emission tomography with [F-18]fluorodeoxyglucose. J Clin Oncol 2006;24:4587-4593.

33. Yan TD, Deraco M, Baratti D. Cytoreductive surgery and hyperthermic intraperitoneal chemotherapy for malignant peritoneal mesothelioma: multi-institutional experience. J Clin Oncol 2009;27: $6237-6242$. 
AQ1: Please confirm whether the inclusion of Short Title is fine.

AQ2: Please confirm that all author names are $\mathrm{OK}$ and are set with first name first, surname last.

AQ3: Please confirm that all affiliation are $\mathrm{OK}$ an are set with order.

AQ4: Please confirm whether the color figures should be reproduced in color or black and white in the print version. If the color figures must be reproduced in color in the print version, please fill the color charge form immediately and return to Production Editor. Or else, the color figures for your article will appear in color in the online version only. 